\title{
Emerging Privacy Issues in Times of Open Science
}

\author{
Benjamin Erb ${ }^{1, *}$, Christoph Bösch ${ }^{1}$, Cornelia Herbert ${ }^{2}$, Frank Kargl ${ }^{1}$, and Christian Montag ${ }^{3}$ \\ ${ }^{1}$ Institute of Distributed Systems, Ulm University, Germany \\ ${ }^{2}$ Department of Applied Emotion and Motivation Psychology, Institute of Psychology and Education, Ulm University, Germany \\ ${ }^{3}$ Department of Molecular Psychology, Institute of Psychology and Education, Ulm University, Germany \\ *Corresponding author: Benjamin Erb, benjamin.erb@uni-ulm.de
}

\begin{abstract}
The open science movement has taken up the important challenge to increase transparency of statistical analyses, to facilitate reproducibility of studies, and to enhance reusability of data sets. To counter the replication crisis in the psychological and related sciences, the movement also urges researchers to publish their primary data sets alongside their articles. While such data publications represent a desirable improvement in terms of transparency and are also helpful for future research (e.g., subsequent meta-analyses or replication studies), we argue that such a procedure can worsen existing privacy issues that are insufficiently considered so far in this context. Recent advances in de-anonymization and re-identification techniques render privacy protection increasingly difficult, as prevalent anonymization mechanisms for handling participants' data might no longer be adequate. When exploiting publicly shared primary data sets, data from multiple studies can be linked with contextual data and eventually, participants can be de-anonymized. Such attacks can either re-identify specific individuals of interest, or they can be used to de-anonymize entire participant cohorts. The threat of de-anonymization attacks can endanger the perceived confidentiality of responses by participants, and ultimately, lower the overall trust of potential participants into the research process due to privacy concerns.
\end{abstract}

Keywords: open science, data publication, privacy, confidentiality

\section{Introduction}

For psychology and other empirical research disciplines, surveys are an essential instrument as they not only provide an easy way to get insights into the inner life of humans (selfdisclosure), but also provide scientists with the chance to run statistical analyses on such data when the questionnaire data has been transformed into categorical, ordinal or metric data. Many disciplines in social and life sciences recently have been shaken by the so-called replication crisis (Shrout \& Rodgers, 2018), in which many prominent findings from literature could not be replicated when research questions were revisited. Such a lack of reproducibility of scientific results challenges empirical work and theoretical models being grounded on non-reproducible work in the first place. Multiple likely causes for the replication crisis have been identified (John, Loewenstein, \& Prelec, 2012; Neuroskep-

Article not yet submitted for publication. Preprint manuscript, version of June 17, 2021.

(ㄷ)(7) This work is licensed under a Creative Commons Attribution 4.0 International License. tic, 2012). Among these are that selection/publication bias favors statistically significant results with solid and intriguing results. These biases create a publishing blindspot for results that are either not statistically significant or inconclusive. The crisis has been aggravated by study results relying on questionable research practices such as HARKing (posthoc hypothesizing; Kerr, 1998) and p-hacking (data dredging for significant outcomes; Head, Holman, Lanfear, Kahn, \& Jennions, 2015), to name only a few.

The Center for Open Science ${ }^{1}$ is an initiative that aims to tackle the aforementioned problems with the Open Science Framework (OSF) ${ }^{2}$. This framework fosters open collaboration in research and improves reproducibility by specifying project workflows, protocols and a set of guidelines established to make each of the research steps taken by a scientist transparent. Beyond, the OSF initiative provides a (pre-)registration platform, preprint servers, as well as data repositories. This allows researchers to (a) preregister a study before it is conducted, (b) to (pre-)publish their findings, and (c) to upload the original data sets statistical analysis is based

\footnotetext{
${ }^{1}$ https://cos.io/

${ }^{2}$ https://osf.io/
} 
on. This not only enables other researchers to verify the statistical analysis, but also to further use these data for metaanalytic purposes (e.g., Foster \& Deardorff, 2017). In the pre-registration process at OSF, scientists need to explain in detail the background of their study, give insights into their hypotheses and power analyses as well as a commitment to a certain kind of statistical analysis to be conducted once the data set has been collected. After data collection, statistical analysis and publication of the paper, the OSF provides the research community with a repository to make the data set underlying a research piece available to all interested scientists. The FAIR guiding principles for scientific data represent a similar initiative (Wilkinson et al., 2016). These principles require scientific data sets to be $f$ indable, acessible, interoperable, and reusable to increase the scientific value of such data sets and to foster secondary usage.

\section{The Role of Privacy in Empirical Research}

Surveys are one main instrument in empirical research to sample individual data points from participants. While there is an intrinsic link between a participant and their responses, the link between the study participants identity and their responses is usually removed through an anonymization step in order to protect participant's privacy. From a research-ethical perspective, such privacy protection has become an imperative mandate within empirical sciences (Folkman, 2000). Besides ethical principles mandating such protection, there are also legal requirements stemming from data protection regulations (e.g., GDPR in European countries). Even further, protection of informational privacy affects the participants' perception of data handling (in particular this is highly relevant, when the research question at hand investigates sensitive topics such as mental disorders, HIV, and so on). Therefore, appropriate privacy protection can improve the participants' trust, and thus improve adherence (Serenko \& Fan, 2013) or prevent detrimental effects such as the social desirability bias in survey-based studies (Joinson, 1999; Krumpal, 2013).

The American Psychological Association (APA) and other professional organizations have acknowledged the relevance of privacy and have added corresponding obligations into their ethical principles. Section four of the APA Ethics Code explicitly addresses privacy and confidentiality. The APA requires confidentiality of collected information as well as a minimization of privacy intrusions. Personally-identifiable information of research participants should not be disclosed without their informed consent. Best practices have been adopted to ensure privacy protection in psychological research. These include the omission of obvious personal identifiers (e.g., real name, social security number) during data collection and the usage of pseudonyms or altered identities for data processing and publishing. Best practices regarding data sharing include disclosure risk assessments, adapted informed consents, and data use agreements for secondary usage (Alter \& Gonzalez, 2018).

The latest recommendations of the German Society for Psychology on the management and provision of psychological research data (Gollwitzer et al., 2021) formulate strict requirements regarding personally identifiable data of participants. This includes the necessity to anonymize or pseudonymize subject data in a way that it prevents a reidentification based on any surveyed subject characteristics - even when these characteristics have been sampled in different studies. Furthermore, the inclusion of participants' data in a data set to be shared with other parties always requires the consent of the participants unless their entries are completely anonymized. This in turn implies that a data entry from a participant can only be part of a published data set if there is an explicit consent for such data publications.

\section{Anonymity in Empirical Research}

While an informed consent given by study participants may satisfy legal requirements, and the removal of obvious identifiers prevents trivial re-identification, we argue that this perspective is typically too short-sighted to address actual ramifications of public data releases in terms of anonymity. Even in the absence of basic personal identifiers, it is often possible to re-identify subjects using available contextual knowledge. Examples for such contextual knowledge include the time frame when the study has been conducted, the location of the authors institutions, the responsible ethics or institutional review board, and the description of the sample and reported recruitment strategyall of which narrow down the likely study participants. Note that these types of metadata are important and unavoidable in the research process. This additional knowledge can turn seemingly insignificant characteristics of a subject into personally identifiable information or at least pseudo-identifiers.

Given a random empirical research study conducted at a psychology department of an arbitrary university, it is likely that psychology students from undergraduate cohorts of that university have participated (due to mandatory subject hours) unless the study imperatively requires a different sample. Due to the WEIRD problem (Arnett, 2008), the study participants will likely be biased towards students with a western, educated, industrialized, rich and democratic background. Having a highly reduced sample population makes it already a lot easier to re-identify entries from a certain individual if there are specific characteristics they can be matched with. Despite the absence of direct identifiers such as names, a set of socio-demographic properties in the data set can provide equally unique fingerprints in the sample. For instance, the combination of belonging to an under-represented age group, gender group, and ethnicity might already be sufficient for re-identification. Depending on the data collected in a study, other data points can yield equally distinctive properties for 
certain subjects (e.g., handedness, physical characteristics). Once the data of a given individual can be re-identified with a certain probability, the full data row can be associated with that individual. This might include highly personal, intimate, and confidential disclosures that the subject has only provided under the assumption of anonymity. As we will show next, such de-anonymization attacks are not only hypothetically feasible. Instead, there is a wide range of actual attacks that have already been executed on different publicly available data sets with confidential personal data.

\section{De-Anonymization Attacks on Data Sets}

De-anonymization attacks are techniques that try to map entries from a given data set to potential matching identities with a possibly high probability. These techniques usually rely on linking entries from the given data set with additionally available external data sets and/or contextual information.

It is known for at least 60 years (Fellegi \& Sunter, 1969; Newcombe, Kennedy, Axford, \& James, 1959; Schlörer, 1975) that a few data points about a data subject, which by themselves are not uniquely identifiable, are collectively equivalent to an identifier. This is especially true for demographic attributes, as Sweeney (2002) has shown in her seminal work in which she identified the medical record of the Governor of Massachusetts from an anonymized data set. More recent approaches claim to potentially reidentify approximately $99,98 \%$ of the US-american population from any data set based on a set of 15 demographic values (Rocher, Hendrickx, \& De Montjoye, 2019).

Nowadays, availability of data is so comprehensive that de-anonymization does no longer rely solely on demographic attributes (Narayanan \& Shmatikov, 2008). Due to the massive amount of data that may contain hundreds or thousands of observations per individual, simple anonymization techniques such as making a few attributes more coarse-grained are not effective against de-anonymization anymore.

Researchers have shown that robust de-anonymization is possible in many domains, such as social networks (Narayanan, Shi, \& Rubinstein, 2011; Narayanan \& Shmatikov, 2009), participants in social media studies (Ayers, Caputi, Nebeker, \& Dredze, 2018), genetic data (Craig, 2016; Ellenbogen \& Narayanan, 2019; Erlich, Shor, Peer, \& Carmi, 2018; Gymrek, McGuire, Golan, Halperin, \& Erlich, 2013; Homer et al., 2008), environmental health studies (Boronow et al., 2020), location data (De Montjoye, Hidalgo, Verleysen, \& Blondel, 2013; Golle \& Partridge, 2009; Zang \& Bolot, 2011), browsing histories (Su, Shukla, Goel, \& Narayanan, 2017), and even writing style (Narayanan et al., 2012). The key finding of all this research, including theoretical evidence (Datta, Sharma, \& Sinha, 2012), is that high-dimensional data is inherently vulnerable to deanonymization.

\section{The Inevitable Problem with Public Data Sharing}

So far, we have briefly discussed that a single data set can be potentially de-anonymized with little additional knowledge. We have also outlined that more advanced deanonymization attack techniques today can facilitate multiple data sets as well as externally collected resources in order to de-anonymize individuals in data sets.

This said, we currently also see the growing willingness towards public data sharing at large scale due to the experiences of the replication crises and new research standards such as those fostered by the Open Science Collaboration (2012, 2015). We argue that large-scale de-anonymization attacks on such shared data sets will eventually pose a real threat for researchers in psychology, their data sets, and especially their study participants. This is because researchers will barely be able to effectively guarantee the anonymity of participants anymore.

The potential risks could be even more substantial, as (a) the possibility of successfully linking a subject increases with the number of data sets the subject is part of, (b) advanced attacks targeting complex psychometric variables are feasible, and (c), researchers cannot protect against future deanonymization attacks to come (in particular with the rapid development of new analysis tools, machine learning, etc.).

The number of different data sets that can be correlated with each other (e.g., originating from the same institution and from a similar time frame) has a strong influence on the success of de-anonymization attacks. Note that additional data sets can have connective properties and fill in missing gaps between existing data sets. For instance, we could think of two existing data sets with mostly distinct values that cannot be linked. Next, we add a third data set that contains sets of values which can be easily linked to both of the former data sets. Eventually, we have a transitive link between all three data sets. We illustrate this by another example: Study A publishes data on Life satisfaction and emotionality, Study B on Driving Anger and personality, and Study C on Life satisfaction and Personality. As Study $\mathrm{C}$ includes both variables appearing in Study A and B, more data points of a person can be brought together. Even if questionnaires have been filled in twice and do not exactly match, in particular trait measures should be stable and machine learning will likely detect such similarity patterns across data sets (also when metric variables are used as in the present example). This means that we now have a number of potential matches where we can link responses from three distinct studies potentially originating from the same participant. Again, this might yield lists of highly personal disclosures which the participant might have not provided in a single survey. In our presented example, one should not forget that usually cornerstone variables such as age and gender are collected. Together with more information on when the study was conducted, where the study was rolled out, etc. de-anonymization is getting more likely. 
A final and often neglected component in the privacy discussion is time. Even if data items were anonymized and published (either intentionally or by mistake) at one point in time when de-anonymization was not (yet) feasible, it is hard to foresee whether data can be fully protected from deanonymization in the future. New data, possibly from other sources, might become available and linkable at some day which will allow the re-identification of data subjects. Besides, new techniques for de-anonymization will likely be found.

\section{Conclusion}

In this paper, we argue that efforts towards more transparency and open science in the aftermath of the replication crisis increase tensions with privacy and data protection and anonymity of study participants is getting more at risk. In emphasizing this, we are not opposing the Open Science Framework or related strategies for improving scientific studies. We fully agree that strategies such as pre-registration, sound power-analysis, transparency about hypotheses, and free sharing of data have a very positive effect on the quality of research conducted. If the participants of a data set have been recruited via the Internet and the publish primary data set only includes rather general information (e.g., coarse information on age, gender and aggregated personality scale values), this will make it at least relatively unlikely that data entries can be mapped back to unique identities. But as soon as data sets contain additional contextual or supplemental information (e.g., timestamps, indirect location information such as geo-locatable IP address fragments), things can change very fast and data sets can become highly sensitive to attacks. These attacks may occur at a later date and could be fueled by cross-linkage to other data sets not yet released and attack schemes not yet developed at the time of the publication. This is an observation that researchers should keep in mind when they release primary data sets to the public.

We advocate to engage in an important discussion to carefully investigate the trade-offs between open science and legal and technical perspectives of data protection. However, we think that having strong data protection and privacy protection does not necessarily mean that transparency and data sharing has to be hindered strongly. Instead, a discussion between the open science and privacy research communities should focus on the question if and how modern forms of privacy-enhancing technologies can be leveraged to implement open science toolkits and platforms with strong privacy protection and data access control mechanisms while at the same time endorsing replicability of results and intended secondary use. Privacy-enhancing technologies could technically enforce necessary types of access control and eventually help bringing together the interests of open science initiatives and researchers as well as the privacy guarantees of research participants.

\section{References}

Alter, G., \& Gonzalez, R. (2018). Responsible practices for data sharing. American Psychologist, 73(2), 146. doi:10.1037/amp0000258

Arnett, J. J. (2008). The neglected 95\%: Why american psychology needs to become less american. American Psychologist, 63(7), 602. doi:10.1037/14805-008

Ayers, J. W., Caputi, T. L., Nebeker, C., \& Dredze, M. (2018). Dont quote me: Reverse identification of research participants in social media studies. NPJ digital medicine, 1(30), 1-2. doi:10.1038/s41746-018-0036-2

Boronow, K. E., Perovich, L. J., Sweeney, L., Yoo, J. S., Rudel, R. A., Brown, P., \& Brody, J. G. (2020). Privacy risks of sharing data from environmental health studies. Environmental Health Perspectives, 128(1), 17008. doi:10.1289/EHP4817

Craig, D. W. (2016). Understanding the links between privacy and public data sharing. nature methods, 13(3), 211-212. doi:10.1038/nmeth.3779

Datta, A., Sharma, D., \& Sinha, A. (2012). Provable deanonymization of large datasets with sparse dimensions. In International Conference on Principles of Security and Trust (pp. 229-248). Springer. doi:10.1007/ 978-3-642-28641-4_13

De Montjoye, Y.-A., Hidalgo, C. A., Verleysen, M., \& Blondel, V. D. (2013). Unique in the crowd: The privacy bounds of human mobility. Scientific reports, 3(1376), 1-5. doi:10.1038/srep01376

Ellenbogen, P., \& Narayanan, A. (2019). Identification of anonymous dna using genealogical triangulation. bioRxiv, 531269. doi:10.1101/531269

Erlich, Y., Shor, T., Peer, I., \& Carmi, S. (2018). Identity inference of genomic data using long-range familial searches. Science, 362(6415), 690-694. doi:10.1126/ science.aau 4832

Fellegi, I. P., \& Sunter, A. B. (1969). A theory for record linkage. Journal of the American Statistical Association, 64(328), 1183-1210. doi:10 . 1080/01621459. 1969. 10501049

Folkman, S. (2000). Privacy and confidentiality. In B. D. Sales \& S. Folkman (Eds.), Ethics in research with human participants (pp. 49-57). American Psychological Association.

Foster, E. D., \& Deardorff, A. (2017). Open science framework (OSF). Journal of the Medical Library Association: JMLA, 105(2), 203.

Golle, P., \& Partridge, K. (2009). On the anonymity of home/work location pairs. In International Conference on Pervasive Computing (pp. 390-397). Springer. doi:10.1007/978-3-642-01516-8_26

Gollwitzer, M., Abele-Brehm, A., Fiebach, C. J., Ramthun, R., Scheel, A., Schönbrodt, F., \& Steinberg, U. (2021). Management und Bereitstellung von Forschungs- 
daten in der Psychologie: Überarbeitung der DGPsEmpfehlungen. Psychologische Rundschau.

Gymrek, M., McGuire, A. L., Golan, D., Halperin, E., \& Erlich, Y. (2013). Identifying personal genomes by surname inference. Science, 339(6117), 321-324. doi:10. 1126/science. 1229566

Head, M. L., Holman, L., Lanfear, R., Kahn, A. T., \& Jennions, M. D. (2015). The extent and consequences of p-hacking in science. PLOS Biology, 13(3), 1-15. doi:10.1371/journal.pbio.1002106

Homer, N., Szelinger, S., Redman, M., Duggan, D., Tembe, W., Muehling, J., ... Craig, D. W. (2008). Resolving individuals contributing trace amounts of dna to highly complex mixtures using high-density snp genotyping microarrays. PLoS Genet, 4(8), e1000167. doi:10 . 1371/journal.pgen.1000167

John, L. K., Loewenstein, G., \& Prelec, D. (2012). Measuring the prevalence of questionable research practices with incentives for truth telling. Psychological science, 23(5), 524-532. doi:10.1177/0956797611430953

Joinson, A. (1999). Social desirability, anonymity, and internet-based questionnaires. Behavior Research Methods, Instruments, $\mathcal{F}$ Computers, 31(3), 433-438. doi:10.3758/BF03200723

Kerr, N. L. (1998). Harking: Hypothesizing after the results are known. Personality and Social Psychology Review, 2(3), 196-217. doi:10.1207/s15327957pspr0203_4

Krumpal, I. (2013). Determinants of social desirability bias in sensitive surveys: A literature review. Quality $\mathcal{E}$ Quantity, 47(4), 2025-2047. doi:10 . 1007/s11135 011-9640-9

Narayanan, A., Paskov, H., Gong, N. Z., Bethencourt, J., Stefanov, E., Shin, E. C. R., \& Song, D. (2012). On the feasibility of internet-scale author identification. In 2012 IEEE Symposium on Security and Privacy (pp. 300-314). doi:10.1109/SP.2012.46

Narayanan, A., Shi, E., \& Rubinstein, B. I. (2011). Link prediction by de-anonymization: How we won the kaggle social network challenge. In 2011 International Joint Conference on Neural Networks (pp. 1825-1834). IEEE. doi:10.1109/IJCNN.2011.6033446

Narayanan, A., \& Shmatikov, V. (2008). Robust deanonymization of large sparse datasets. In 2008 IEEE Symposium on Security and Privacy (pp. 111-125). doi:10.1109/SP.2008.33

Narayanan, A., \& Shmatikov, V. (2009). De-anonymizing social networks. In 2009 IEEE Symposium on Security and Privacy (pp. 173-187). doi:10.1109/SP.2009.22

Neuroskeptic. (2012). The nine circles of scientific hell. Perspectives on Psychological Science, 7(6), 643-644. doi:10.1177/1745691612459519
Newcombe, H. B., Kennedy, J. M., Axford, S. J., \& James, A. P. (1959). Automatic linkage of vital records. Science, 130(3381), 954-959. doi:10.1126/science.130. 3381.954

Open Science Collaboration. (2012). An open, largescale, collaborative effort to estimate the reproducibility of psychological science. Perspectives on Psychological Science, 7(6), 657-660. doi:10 . 1177 / 1745691612462588

Open Science Collaboration. (2015). Estimating the reproducibility of psychological science. Science, 349(6251). doi:10.1126/science.aac4716

Rocher, L., Hendrickx, J. M., \& De Montjoye, Y.-A. (2019). Estimating the success of re-identifications in incomplete datasets using generative models. Nature communications, 10(3069), 1-9. doi:10.1038/s41467-01910933-3

Schlörer, J. (1975). Identification and retrieval of personal records from a statistical data bank. Methods of Information in Medicine, 14(01), 7-13. doi:10.1055/s0038-1635690

Serenko, N., \& Fan, L. (2013). Patients perceptions of privacy and their outcomes in healthcare. International Journal of Behavioural and Healthcare Research, 4(2), 101-122. doi:10.1504/IJBHR.2013.057359

Shrout, P. E., \& Rodgers, J. L. (2018). Psychology, science, and knowledge construction: Broadening perspectives from the replication crisis. Annual Review of Psychology, 69(1), 487-510. doi:10 . 1146/ annurev - psych 122216-011845

Su, J., Shukla, A., Goel, S., \& Narayanan, A. (2017). Deanonymizing web browsing data with social networks. In Proceedings of the 26th International Conference on World Wide Web (pp. 1261-1269). doi:10.1145/ 3038912.3052714

Sweeney, L. (2002). K-anonymity: A model for protecting privacy. International Journal of Uncertainty, Fuzziness and Knowledge-Based Systems, 10(05), 557-570. doi:10.1142/S0218488502001648

Wilkinson, M. D., Dumontier, M., Aalbersberg, I. J., Appleton, G., Axton, M., Baak, A., ... Bourne, P. E., et al. (2016). The fair guiding principles for scientific data management and stewardship. Scientific data, 3(1), 19. doi:doi.org/10.1038/sdata.2016.18

Zang, H., \& Bolot, J. (2011). Anonymization of location data does not work: A large-scale measurement study. In Proceedings of the 17th Annual International Conference on Mobile Computing and Networking (pp. 145156). doi:10.1145/2030613.2030630 